\title{
X-rays from young star clusters: a complement to optical and infrared views
}

\author{
Francesco Damiani \\ INAF - Osservatorio Astronomico di Palermo, \\ Piazza del Parlamento 1, 90134 Palermo, Italy \\ email: damiani@astropa.inaf .it
}

\begin{abstract}
In the last few years, X-ray observational studies of young star clusters have advanced significantly, mainly thanks to the great capabilities of current X-ray observatories such as Chandra and XMM/Newton. In addition to enabling a detailed study of coronae of individual bright stars, high-spatial-resolution X-ray observations of many young clusters and star-forming regions, even massive and distant ones, have led to the detection of large populations of X-ray-bright members, often down to subsolar masses, and despite strong absorption. The peculiar ability of X-ray emission to select young, low-mass cluster stars against a crowded Galactic-plane field-star background has permitted better studies of global cluster properties, with respect to optical/infrared studies alone, including of cluster initial mass functions (across wide mass ranges), star-formation histories (with indication of age spreads - or even sequencesin many clusters) and morphologies (various degrees of symmetry and dynamical relaxation), sometimes with evidence of mass segregation. Also, the complementary availability of X-ray and optical/infrared data has enabled to place constraints on lifetimes and depletion mechanisms of pre-main-sequence circumstellar disks.
\end{abstract}

Keywords. stars: pre-main-sequence, open clusters and associations: general, X-rays: stars

\section{Introduction: $\mathrm{X}$-ray emission of young stars}

X-ray observations have become an important tool to study stellar clusters, especially the youngest ones, including star-formation regions (SFRs). This is related to the properties of X-ray emission of young stars, which have been the subject of extensive reviews elsewhere (e.g., Feigelson \& Montmerle 1999; Güdel 2004; Feigelson et al. 2007; Güdel \& Nazé 2009). Very briefly, X-ray emission from solar-type stars was found to decrease strongly with stellar age, being highest at the earliest stellar evolutionary stages, during the pre-main-sequence (PMS) phase. This result was already found very clearly from the earliest studies of open clusters (Pleiades, Hyades) and SFRs (Taurus-Auriga, Chamaeleon) done with the Einstein observatory (see, e.g., Damiani et al. 1995; and references therein). More recent, deeper X-ray data have yielded slightly different (lower) mean X-ray luminosities, $L_{\mathrm{X}}$, for some star clusters or groups, but the decrease with age is clearly still there. Therefore, very young (PMS) stars stand out clearly in X-ray images with respect to older field stars, which are $\sim 1000$ times fainter. One decade after the Einstein observations, X-ray data obtained with the ROSAT satellite also permitted to discover genuine new clusters, such as those around $\sigma$ Ori (Walter et al. 1997) or $\eta$ Cha (Mamajek et al. 1999).

\section{XMM-Newton and Chandra observations of young clusters}

With respect to Einstein and ROSAT, the much better sensitivity, spatial and spectral resolution of the newest generation of X-ray space observatories with good imaging 
capabilities, XMM-Newton and Chandra†, both operating since late 1999-early 2000, have permitted significant advances in X-ray studies of young clusters. Both the number of SFRs studied, and the amount of detailed X-ray data which have become available for each SFR, have grown considerably.

To better appreciate the amount of new X-ray observational data now available for such clusters, and the many projects making use of them, often very recent or still ongoing, Tables 1 and 2 report lists of all young clusters observed to date by XMM-Newton and Chandra, respectively. In these lists, observations of small PMS star groups (e.g., near Herbig Ae/Be stars) or observations of individual PMS stars are not included. A number of the listed papers, moreover, are not studies of the properties of the entire population of a cluster, but only of some selected members, and therefore they will not be discussed further.

Clearly, the Chandra cluster list is nearly twice as long as the XMM-Newton list. It also includes a larger percentage of giant SFRs, often at large distances (say, > $1.5 \mathrm{kpc}$ ), hosting rich and crowded stellar populations: in these cases, the very high spatial resolution offered by Chandra images (PSF $\sim 0.5^{\prime \prime}$ FWHM on-axis) is really needed to spatially resolve the clusters' stellar X-ray sources, while XMM-Newton images (PSF $\sim 4-5^{\prime \prime}$ FWHM) of such clusters are often affected by source confusion, with many of the fainter sources being missed, and are more useful for X-ray studies of bright, massive cluster stars. As an example, NGC 6231 ( $d \sim 1.3 \mathrm{kpc}$ ) was observed using both the XMMNewton EPIC (170 ks exposure; $\sim 800$ detected sources in a $\sim 30^{\prime} \times 30^{\prime}$ field of view) and Chandra ACIS instruments (120 ks exposure; $\sim 1500$ sources in a $\sim 17^{\prime} \times 17^{\prime}$ field). Despite the shorter exposure time and smaller field of view, Chandra has detected almost twice as many sources as XMM-Newton.

\subsection{X-rays versus other selection methods}

It may be useful to give some rough numbers on the relative efficiency in selecting various types of young stars, by using different methods, as it emerges from the literature listed in Tables 1 and 2:

- X-rays: up to $90 \%$ success rate (especially for rich clusters; effective for Class I-III stars).

- Infrared (IR)/H $\alpha$ emission: widely variable 5-70\% success rate (but useful for objects belonging to Classes I-II only, since it misses Class III stars).

- Proper motion: in principle, a better membership indicator, but available only for relatively few stars in not many clusters.

- Lithium (plus radial velocity) test: 'definitive' test, but observing with sufficiently high spectral resolution over 10000 stars in a typical $20^{\prime} \times 20^{\prime}$ Galactic-plane cluster field down to, e.g., $V=20$ mag takes a very long time.

An example of a high-mass SFR, studied in detail at many wavelengths including Xrays, is the cluster NGC 6530, in the HII region M8 (Lagoon Nebula). This is a typical 'blister' SFR, where the massive star cluster NGC 6530 excavates a cavity in the molecular cloud. For this cluster, sequential star formation was suggested by Lada et al. (1976). The Chandra ACIS images of this region show a complex structure (Figure 1), with the bulk of the cluster members found in a central, older, almost spherical cluster (Damiani et al. 2004), and younger subclusters of stars lying both to the west (around the wellknown O7.5 star Herschel 36, embedded in the Hourglass Nebula) and to the south-east (near the massive young stellar object M8-East IR). About 1500 X-ray point sources are

$\dagger$ Other X-ray observatories have observed PMS stars between the ROSAT and XMM-Newton/Chandra eras, such as ASCA and BeppoSAX; their results are, however, more relevant for studies of coronal physics, not for cluster studies, and will not be discussed here. 
Table 1. XMM-Newton observations of young clusters

\begin{tabular}{|c|c|}
\hline Taurus-Auriga & $\begin{array}{l}\text { Many papers, e.g., Favata et al. }(2003) \text {; } \\
\text { XEST project: Güdel et al. }(2007) \text {; new members in Scelsi et al. (2008) }\end{array}$ \\
\hline$\rho \mathrm{Oph}$ & Ozawa et al. (2005); Giardino et al. (2007) \\
\hline Lupus & Gondoin (2006) \\
\hline Upper Sco-Cen & Argiroffi et al. (2006) \\
\hline Cha I & Stelzer et al. (2004); Robrade \& Schmitt (2007) \\
\hline TW Hya & Stelzer \& Schmitt (2004); Argiroffi et al. (2005) \\
\hline $\mathrm{CrA}$ & Hamaguchi et al. (2005); Forbrich et al. (2006) \\
\hline NGC1333 & Preibisch (2003) \\
\hline IC 348 & Preibisch \& Zinnecker (2004) \\
\hline IC 2391 & Marino et al. (2005) \\
\hline IC 2602 & - \\
\hline NGC 2547 & Jeffries et al. (2006) \\
\hline Serpens & Preibisch (2003) \\
\hline 25 Ori & - \\
\hline Barnard $30 / 35$ & - \\
\hline L1251 & Simon $(2006)$ \\
\hline Barnard 335 & - \\
\hline $\mathrm{OMC} 2 / 3$ & - \\
\hline$\sigma$ Ori & Franciosini et al. (2006) \\
\hline$\lambda$ Ori & - \\
\hline NGC2024 & - \\
\hline NGC2023 & - \\
\hline NGC2071 & Skinner et al. (2007) \\
\hline NGC2264 & Simon \& Dahm (2005) \\
\hline IC 5146 & Skinner et al. (2009) \\
\hline NGC6383 & Rauw et al. (2003) \\
\hline$\gamma^{2}$ Vel & Jeffries et al. (2009) \\
\hline NGC 2362 & - \\
\hline NGC6530 & Rauw et al. (2002) \\
\hline NGC6231 & Sana et al. $(2006,2007)$ \\
\hline NGC6604 & De Becker et al. (2005) \\
\hline M17 & - \\
\hline Cyg OB2 & Linder et al. (2009) \\
\hline$\chi$ Per (NGC884) & - \\
\hline $\operatorname{Tr} 16 / 14 / \mathrm{Car} \mathrm{OB} 1$ & Albacete Colombo et al. (2003); Antokhin et al. (2008) \\
\hline Westerlund 1 & Muno et al. (2007) \\
\hline
\end{tabular}

detected, down to about $0.3 \mathrm{M}_{\odot}$. Deep follow-up optical photometry (Prisinzano et al. 2005 ) obtained with WIFI (at the ESO $2.2 \mathrm{~m}$ telescope) has shown a well-defined X-rayselected cluster sequence down to $V \sim 21 \mathrm{mag}$, which has been used to compute ages, masses and the initial mass function (IMF; see next section). A VLT/Giraffe spectroscopic study of radial velocity/lithium (Prisinzano et al. 2007) yielded an X-ray selection completeness of $\geqslant 90 \%$ down to $V \sim 18$ mag.

\section{Recent results}

The many new observational studies listed in Tables 1 and 2 have provided important information on the stellar populations in those clusters. In most instances, the bulk of the cluster members (often many hundreds) were selected individually for the first time, with little or no confusion, and reliable optical/IR identifications with very few $(<1 \%)$ ambiguous cases. This permitted to address a number of questions, which I outline here.

\subsection{Cluster morphologies}

Detection of a sizable population of a cluster permits to study its morphology, which may offer clues as to the cluster's dynamical state: quasi-spherical clusters are likely relaxed dynamically, while those with an irregular distribution of stars are dynamically less evolved. Very few young clusters are actually spherical, and those with the most regular morphologies still show an asymmetry of some sort. In this category should be placed, e.g., NGC 2362, NGC 6231, NGC 6611, W3 Main, NGC 2244 and the Orion Nebula Cluster (ONC), to a limited extent. 
Table 2. Chandra observations of young clusters

\begin{tabular}{|c|c|}
\hline Taurus-Auriga & Many papers on individual stars \\
\hline$\eta$ Cha & Evans et al. $(2003,2004)$ \\
\hline$\varepsilon$ Cha & Feigelson et al. (2003); Testa et al. (2008) \\
\hline Cha I & Feigelson \& Lawson (2004) \\
\hline $\mathrm{CrA}+$ Coronet cluster & Hamaguchi et al. (2005); Forbrich et al. (2007) \\
\hline$\rho \mathrm{Oph}$ & Imanishi et al. (2001); Gagné et al. (2004) \\
\hline TW Hya association & Gizis et al. (2004) \\
\hline NGC1333 & Getman et al. (2002) \\
\hline IC 348 & Preibisch \& Zinnecker $(2001,2002)$ \\
\hline $\mathrm{L} 1251 \mathrm{~B}$ & Simon $(2009)$ \\
\hline L $1448 \mathrm{~N}-\mathrm{A}$ & Tsujimoto et al. (2005) \\
\hline L1415 & - \\
\hline L1527 & - \\
\hline $\mathrm{LkH} \alpha 101$ & Osten \& Wolk (2009) \\
\hline Orion Nebula Cluster & $\begin{array}{l}\text { Garmire et al. }(2000) \text {; Flaccomio et al. }(2003 \mathrm{a}, \mathrm{b}) \text {; Feigelson et al. }(2003) \text {; } \\
\text { COUP project: Getman et al. }(2005) \text {, and entire } A p J S \text { vol. } 160\end{array}$ \\
\hline Orion flanking fields & Ramirez et al. (2004); Rebull et al. (2006) \\
\hline M78/NGC2068 & Grosso et al. (2004) \\
\hline NGC2071 & Skinner et al. (2009) \\
\hline NGC2024 & Skinner et al. (2003) \\
\hline OMC $2-3$ & Tsuboi et al. (2001); Tsujimoto et al. (2002) \\
\hline$\lambda$ Ori & - \\
\hline$\sigma$ Ori & Skinner et al. (2008) \\
\hline L1630 (HH 24-26) & Simon et al. (2004) \\
\hline Mon R2 & Kohno et al. (2002); Nakajima et al. (2003) \\
\hline NGC2244 (Rosette) & Townsley et al. (2003); Wang et al. (2008, 2009) \\
\hline NGC2264 North/South & Ramirez et al. (2004); Rebull et al. (2006); Flaccomio et al. (2006) \\
\hline NGC2362 & Damiani et al. (2006); Delgado et al. (2006) \\
\hline NGC7129 & Stelzer \& Scholz (2009) \\
\hline IC $1396-\mathrm{N}$ & Getman et al. (2007) \\
\hline CG12 & Getman et al. (2008) \\
\hline h Per & Currie et al. (2009) \\
\hline DB2001CL-123 & - \\
\hline NGC281 & - \\
\hline Cep B & Getman et al. (2006) \\
\hline M8/NGC6530 & Damiani et al. (2004) \\
\hline M20/NGC6514 & Rho et al. (2004) \\
\hline M16/NGC6611 & Linsky et al. (2007); Guarcello et al. (2007) \\
\hline M17/NGC6618 & Townsley et al. (2003); Broos et al. (2007) \\
\hline NGC6231 & Damiani et al. (2009) \\
\hline NGC6334 & Ezoe et al. (2006); Feigelson et al. (2009) \\
\hline NGC6357 & Wang et al. (2007) \\
\hline RCW 38 & Wolk et al. (2006) \\
\hline W3 & Hofner et al. (2002); Feigelson \& Townsley (2008) \\
\hline W 51 & Koo et al. (2005) \\
\hline Cyg OB2 & Albacete Colombo et al. (2007) \\
\hline S106 & Giardino et al. (2004) \\
\hline Berkeley 87 & - \\
\hline Serpens & Giardino et al. (2007); Winston et al. (2007) \\
\hline RCW 36 & - \\
\hline W $49 \mathrm{~A}$ & Tsujimoto et al. (2006) \\
\hline NGC7538 & - \\
\hline IC 1805 & - \\
\hline Sh2-187 & - \\
\hline NGC1893 & Caramazza et al. (2008) \\
\hline DR 21 & - \\
\hline W $75 \mathrm{~N}$ & - \\
\hline GGD 27 & Pravdo et al. (2009) \\
\hline W3 (Main/North/OH) & Feigelson \& Townsley (2008) \\
\hline RCW $108 /$ NGC6193 & Skinner et al. (2005); Wolk et al. (2008) \\
\hline Trumpler 14 & Townsley $(2006)$ \\
\hline Trumpler 16 & Evans et al. (2004); Albacete Colombo et al. (2008) \\
\hline Carina complex & - \\
\hline NGC3576 & Townsley (2006) \\
\hline NGC3603 & Moffat et al. (2002); Poteet et al. (2004) \\
\hline Westerlund 1 & Skinner et al. (2006); Clark et al. (2008) \\
\hline RCW 49 (Westerlund 2) & Tsujimoto et al. (2007); Nazé et al. (2008) \\
\hline Sgr B2 & Takagi et al. (2002) \\
\hline Arches & Yusef-Zadeh et al. (2002); Wang et al. (2006) \\
\hline RSG 2 & - \\
\hline
\end{tabular}




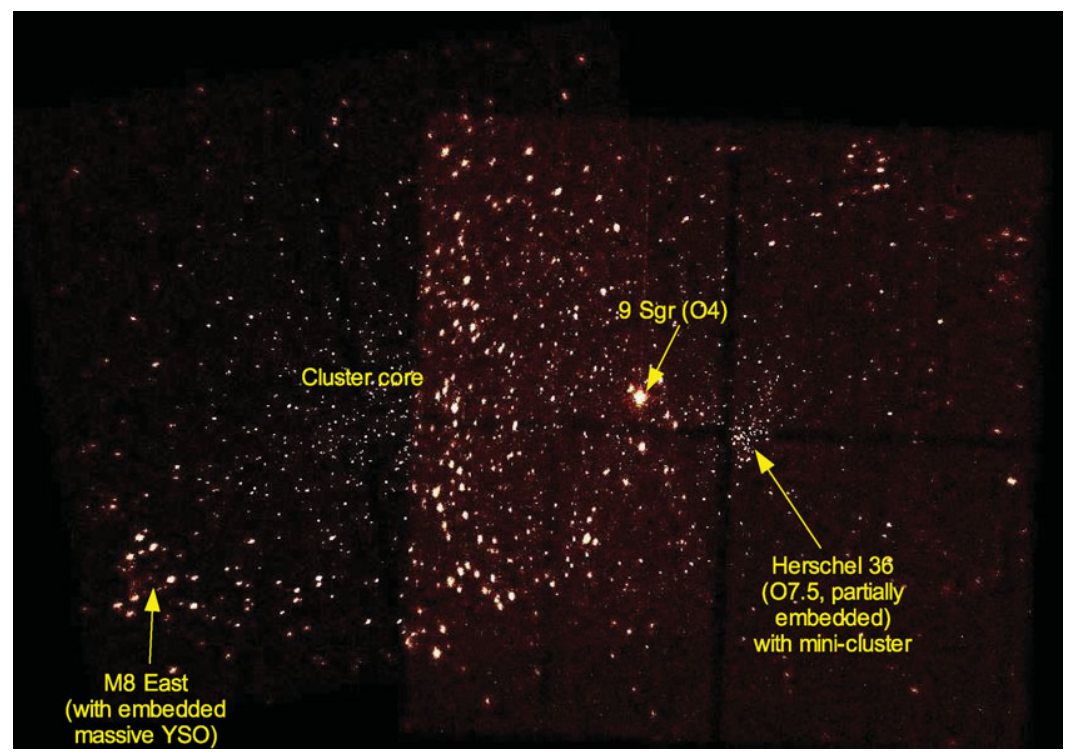

Figure 1. NGC 6530 and M8 in X-rays. Chandra ACIS mosaic $\left(\sim 20^{\prime} \times 30^{\prime}\right)$.

Many other clusters instead show either an elongated, irregular shape, or even multiple star-forming subclusters, which can be taken as indicative that stars in each subcluster have not traveled very far since their formation, and are thus still near their birth places. Examples include NGC 6530/M8, NGC 6618/M17, Cyg OB2, NGC 6334, NGC 1893, W3 $(\mathrm{OH})$ and Cep B.

\subsection{Cluster subpopulations}

The X-ray discovery of individual cluster members across a wide range of masses and extinctions (and, by inference, PMS evolutionary stage) permits comparison of different subpopulations in some clusters.

Low-mass versus massive stars: The low-mass stellar density peaks usually around OB stars. This is true in both near-spherical and clumpy clusters, where (often) each clump has one (or more) massive star(s) at its center. However, sometimes O stars are found off stellar-density peaks: examples are $\eta$ Car in Trumpler 16, 9 Sgr in NGC 6530, HD 46223 in NGC 2244, the O star in W3 North (Albacete Colombo et al. 2008; Damiani et al. 2004; Wang et al. 2008, 2009; Feigelson \& Townsley 2008). The reason of these 'anomalies' is still unknown: they are perhaps caused by dynamical evolution (runaway stars), or are signs of a different mode of massive-star formation.

Obscured/embedded versus unobscured stars: Cluster subpopulations characterized by large soft-X-ray absorption, indicative of heavy obscuration $\left(n_{\mathrm{H}} \geqslant 10^{22} \mathrm{~cm}^{-2}\right)$ are probably embedded, very young populations. They are often spatially distinct from more exposed $\left(n_{\mathrm{H}} \leqslant 10^{21} \mathrm{~cm}^{-2}\right)$ subpopulations in the same cluster: this was found in, e.g., NGC 6334, Cep B, NGC 6618 and NGC 6357 and is probably an indication of different episodes of star formation in the same region (Feigelson et al. 2009; Getman et al. 2006; Broos et al. 2007; Wang et al. 2007).

The nature of the obscured X-ray-selected cluster members is not easily assessed on the basis of available photometry in the (near-) IR (these stars are usually undetected in optical bands). A typical 2MAss color-magnitude diagram (CMD) of such X-ray-selected stars shows their strong and nonuniform extinction, and that the identification of a 
cluster sequence would be extremely hard on the basis of the IR data alone: for such a cluster population, X-ray membership information is evidently crucial. Moreover, the newly selected members from X-rays are not confined to the low-mass part of the cluster population: from de-reddening the positions in the IR CMD one also finds new massive candidate cluster members, e.g., in NGC 6618 (Broos et al. 2007) or NGC 6193 (Wolk et al. 2008), which look like very red objects (and were overlooked in previous studies) because of substantial extinction. In similar cases, unambiguous determinations of stellar masses/ages are impossible based on photometry alone, or at best highly uncertain.

\subsection{Age spread/sequences}

The study of X-ray-selected populations has also permitted to find differences in starformation histories between clusters (based on isochronal stellar ages when feasible, or using other age indicators):

- Formation during an extended period of time (5-10 Myr): e.g., NGC 6530 (Damiani et al. 2004; Prisinzano et al. 2005), NGC 6231 (Damiani et al. 2009);

- Nearly simultaneous formation (within 1 Myr): NGC 2362 (Damiani et al. 2006; in agreement with the previous study by Moitinho et al. 2001);

- Formation along mass sequences: e.g., NGC 6231 (low-mass stars 10 Myr ago, massive stars $\sim 2$ Myr ago; Damiani et al. 2009), W3 Main (low-mass stars $\sim 1 \mathrm{Myr}$ ago, massive stars still in ultracompact HiI region, $\leqslant 0.1$ Myr ago; Feigelson \& Townsley 2008).

These studies complement, therefore, similar studies of cluster stellar ages in nearer, better-studied clusters such as Taurus-Auriga and Orion (of lower masses, however), with comparable results.

\subsection{Triggering}

In a few cases, the characteristics of the X-ray-detected population in a SFR have been found compatible with those expected for triggered star formation by one or more massive stars. One piece of evidence in favor of this hypothesis is star formation through spatial sequences, with spatial subgroups having different average ages, as derived from either location in the CMD, varying morphology (relaxed versus knotty), differing amount of absorption or other youth indicators (disks, ultracompact HiI regions, etc.).

Examples include NGC 6530/M8, where both isochronal ages and the evolutionary stages of the objects suggest a time sequence from the older cluster core, to the younger Hourglass Nebula, to the youngest M8 East IR protostar (Damiani et al. 2004; supporting earlier suggestions by Lada et al. 1976). Another example is the Cep B/Cep OB3b/Sh 155 complex (Getman et al. 2006, 2009), where the suggested sequence runs from northwest (outside the HII region Sh 155, older stars) to southeast (inside Sh 155, younger embedded cluster), on the basis of both the degree of X-ray absorption and disk frequency (both proxies for the stellar evolutionary stage). The two youth indicators agree with one another, and their spatial location also agrees qualitatively with the comet-head shape of Sh 155, and the position of the massive ionizing O7 star HD 217086, which the head of the Sh 155 nebula points to.

Finally, Getman et al. (2007) have found evidence of triggering also in the IC $1396 \mathrm{~N}$ globule. Here, a spatial age sequence is found as a string of embedded Class I/0 objects (inside the globule), Class II (midway), and X-ray-selected Class III stars (outside the globule towards the exciting star HD 206267). This agrees with a radiation-driven implosion (RDI) model (e.g., Lefloch \& Lazareff 1994) for triggered star formation in bright-rimmed clouds. On the other hand, the same authors (Getman et al. 2008) find that the simple RDI model is not compatible with their Chandra X-ray observation of 
another small star-forming globule, CG 12, where many distinct episodes of triggering might have taken place instead.

\subsection{Initial mass function}

The IMF has been studied intensively for many clusters, benefitting from the relatively easy and unbiased selection of large populations of members using X-ray data. Despite having been studied for decades, important questions still remain: Is the IMF universal? If not, how does it depend on ambient conditions? Are there clusters with top-heavy IMFs?

Different X-ray-based methods have been devised to address these questions: (a) Find optical counterparts to X-ray sources (especially low-mass; massive stars have often better-characterized membership), derive their masses from the position in the CMD and PMS evolutionary models (when feasible) and make a mass histogram. (b) Alternatively, compare the hard-band (less sensitive to absorption) X-ray luminosity function with that of well-studied, template clusters (e.g., the ONC), test shape similarity and normalize. Of course, these procedures are not free of uncertainties, and a few warnings are in order to obtain reliable results:

1. An X-ray IMF determination must be corrected for X-ray (in)completeness, especially in lowest-mass bins (e.g., Prisinzano et al. 2005 for NGC 6530).

2. If many cluster stars have IR excesses, photometrically derived masses are likely inaccurate, and one must resort to spectroscopy (Prisinzano et al. 2007; again for NGC $6530)$.

3. If many optically/IR unidentified X-ray sources remain (which still show clear spatial clustering, and are thus mostly members) one can try to include them by deriving stellar masses from X-ray luminosities, $L_{\mathrm{X}}$, using the 'saturation' relation for low-mass stars $L_{\mathrm{X}} \sim 10^{-3.5} L_{\mathrm{bol}}$ (e.g., Randich et al. 2000; Flaccomio et al. 2003) and the $L_{\mathrm{bol}}{ }^{-}$ mass relation appropriate to the cluster age/reddening (e.g., Damiani et al. 2009: NGC 6231).

4. Optical glare from OB stars may prevent finding faint, low-mass stars as counterparts to detected X-ray sources: it is much less so in X-rays, since $L_{\mathrm{X}} / L_{\mathrm{bol}} \sim 10^{-3.5}$ for low mass stars, but $L_{\mathrm{X}} / L_{\mathrm{bol}} \sim 10^{-7}$ for OB stars (Pallavicini et al. 1981). Therefore, the brightness contrast is reduced from $\sim 10^{6}$ in the optical to only $10^{2}-10^{3}$ at X-rays, which are thus an especially good tool to pinpoint low-mass stars near bright and massive ones in a dense cluster (e.g., NGC 2244: Wang et al. 2009).

Results of studies of cluster IMFs include, for many clusters, the first IMF determination, often ranging down to (sub)solar-mass stars: up to 2 orders of magnitude in mass may be covered (e.g., NGC 6231: Damiani et al. 2009). A comparison between the IMFs of many clusters, derived using X-ray data, shows that the upper part of the IMF is generally found to be consistent with a Salpeter IMF. A turnoff is found at some mass, generally subsolar (but NGC 2362 is a possible exception; Damiani et al. 2006). However, the turnoff mass appears to be slightly different from cluster to cluster.

A comparison with a previous optical/H $\alpha$ IMF determination for NGC 6231 (Sung et al. 1998) shows that this latter misses weak-line T Tauri stars entirely (90\% of the low-mass stars in this case), yielding an apparent deficit of low-mass stars in this cluster. The rich, low-mass population of NGC 6231 is instead clearly detected in the X-ray data (Damiani et al. 2009).

X-ray luminosity functions (XLFs) have also been computed for many high-mass clusters and are another useful tool to examine possible IMF differences from cluster to cluster. Analogously to IMFs, XLFs of different massive young clusters are generally similar (see Wang et al. 2008 for a comparison among NGC 6357, NGC 2244, Cep B, 
Table 3. Circumstellar-disk frequencies

\begin{tabular}{lllll}
\hline Cluster & Age $($ Myr $)$ & Diagnostics & Mass range & Frequency \\
\hline ONC & 1 & near-IR & $M>1 \mathrm{M}_{\odot}$ & $20.6 \%$ \\
Cyg OB2 & 2 & near-IR & $M>1 \mathrm{M}_{\odot}$ & $4.4 \%$ \\
NGC2264 & 3 & H $\alpha$ & & $42 \%$ \\
NGC1893 & $3-4$ & Spitzer SED & & $67 \%$ \\
NGC2362 & 5 & H $\alpha$ (IR) & & $5 \%(12 \%)$ \\
NGC6530 & $2-3$ & near-IR & $M>0.5 \mathrm{M}_{\odot}$ & $20 \%$ \\
NGC6611 & 1 & near-IR & $M>0.5 \mathrm{M}_{\odot}$ & $20 \%$ \\
Tr 16 & 3 & near-IR & $M>0.5-1 \mathrm{M} \odot$ & $15 \%$ \\
NGC6231 & $5-7$ & near-IR & $M>1 \mathrm{M} \odot$ & $4 \%$ \\
\hline
\end{tabular}

(1): The frequency becomes higher using more indicators/a larger mass range.

ONC and M17 XLFs). When scaling the observed $L_{\mathrm{X}}$ distributions to that of the ONC, no low-mass-star deficit is found. There appears to be no top-heavy IMF in any of the clusters studied by this method.

\subsection{Mass segregation}

$\mathrm{X}$-ray data also permit to address the issue of mass segregation in high-mass clusters. The question is well posed only for those clusters with approximate spherical shape, where a meaningful center exists. Here, the X-ray-traced low-mass population can be compared to the (usually optically selected) massive stars.

Mass segregation has been found in a few X-ray-studied clusters (e.g., NGC 6231: Damiani et al. 2009; NGC 2362: Damiani et al. 2006), while it has not been found in others (e.g., NGC 2244: Wang et al. 2008, 2009). Whether this difference is due to dynamical evolution, or it is of primordial origin, remains unknown.

\subsection{Disk frequency versus age and environment}

Since different diagnostics of the presence and type of PMS stellar disks (inner disk from near-IR, outer disk from far-IR), or of classical T Tauri star status (accretion through $\mathrm{H} \alpha$, optical/ultraviolet veiling) are found in the literature, it is not always possible to use a uniform disk indicator for a comparison of disk frequencies among many clusters. Using X-ray-selected diskless members, and various disk/accretion indicators, Table 3 compares the circumstellar-disk frequency (in a selected mass range) for several clusters.

The low disk frequencies found in Cyg OB2 (Albacete Colombo et al. 2007) and perhaps NGC 6231 (Damiani et al. 2009) are possibly caused by fast disk evolution under the effect of the radiation field of $\mathrm{OB}$ stars (photoevaporation). Another clue to the same effect has been found in NGC 6611 by Guarcello et al. (2007): disks are significantly less frequent in regions in which the OB stars' integrated ultraviolet flux is more intense, within the same cluster. This suggests strongly that age is not the only relevant parameter for disk evolution.

\subsection{Diffuse X-ray emission}

Genuinely diffuse X-ray emission from hot gas has been found in X-ray images of several high-mass clusters (Orion Nebula: Güdel et al. 2008; NGC 2024: Ezoe et al. 2006; M17: Townsley et al. 2003; Rosette: Wang et al. 2009; Westerlund 1: Muno et al. 2006; Carina Nebula: Ezoe et al. 2009; RCW38: Wolk et al. 2002, 2006; NGC 6334: Ezoe et al. 2006; 30 Dor in the LMC: Townsley et al. 2006), in excess of the extrapolated contribution of weak, unresolved point sources, often in a different location and with a different X-ray spectrum than the point sources.

The favored picture for the origin of diffuse X-rays envisages that winds from massive stars collide and coalesce. Then, a 'champagne flow' of hot gas flows out of the opening 
in the blister molecular cloud. Thus, a molecular cloud is no longer a cold place once massive stars form.

The X-ray spectrum of such diffuse X-ray emission is often soft (in Orion, M17, Rosette, 30 Dor); sometimes, a hard spectral component is also present (NGC 2024, RCW38, Westerlund 1), and a complex spatial/spectral structure was found in the giant molecular complexes of Carina and NGC 6334.

\subsection{The largest young clusters in our Galaxy in X-rays}

Most stars in the Galaxy form in large clusters, containing $>10000$ stars, rather than in 'smallish' SFRs like Taurus-Auriga, with $\sim 100-200$ members. The study of stellar formation (and early evolution) in such giant clusters is therefore very important to understand the condition at birth of the 'average' stars in the Galaxy. However, the largest clusters in the Galaxy lie mostly at large distances, and behind considerable Galactic-plane absorption, which make their rich (and crowded) low-mass population hard to study. Three of such Galactic clusters have been studied in X-rays with Chandra:

- Westerlund 1 (mass $\sim 10^{5} \mathrm{M}_{\odot}$, age $\sim 4-5 \mathrm{Myr}$, distance $\sim 5 \mathrm{kpc}$ ): two exposures of $18+42 \mathrm{ks}$ have detected $46 \mathrm{O}$ and Wolf-Rayet (WR) stars, diffuse X-ray emission and 45 candidate PMS stars, out of an inferred population of $>32000$ low-mass PMS stars (Clark et al. 2008).

- Westerlund $2\left(R C W_{4} 9\right)$ (age $\sim 2 \mathrm{Myr}$, uncertain distance in the range 2-8 kpc (!)): hosts 12 known early-O stars, 2 WR stars, including WR20a $\left(82+83 \mathrm{M}_{\odot}\right)$. One exposure of $40 \mathrm{ks}$ has detected more than 350 new candidate low-mass (PMS) members and also 30 candidate OB members. Possible triggered star formation has been suggested in the southeastern region (Tsujimoto et al. 2007; Nazé et al. 2008).

- $N G C 3603$ (age $\sim 0.3-1 \mathrm{Myr}$, distance $\sim 7 \mathrm{kpc}$ ): analogous to starburst clusters in, e.g., M82. One exposure of $50 \mathrm{ks}$ has detected 348 X-ray sources, of which 40 are OB and WR stars, and most of the others are candidate PMS members (Moffat et al. 2002).

In these clusters, current X-ray data have only detected a minor fraction of the (estimated) low-mass cluster population, because of the insufficient depth of the observations, and deeper observations capable of detecting the bulk of the cluster stars and pushing to their limits the capabilities of current X-ray instruments would be highly desirable.

\section{Large projects with focus on star-formation regions}

A number of recent or ongoing projects of X-ray studies of SFRs exist, based on X-ray observations, either very deep exposures or covering thoroughly a given region.

- Coup: 'Chandra Orion Ultradeep Project' (PI E. Feigelson): a 850 ks ( 10 days) nearly uninterrupted (spacecraft-operations permitting) deep pointing of the Orion Nebula Cluster, presented by Getman et al. (2005); all of ApJS vol. 160 was devoted to the project. In total, $1616 \mathrm{X}$-ray sources were found (hundreds of them heavily obscured), as well as 75 new cluster members.

- Xest: 'X-ray Extended Survey of Taurus' (PI M. Güdel): a survey with XMMNewton, using more than 20 medium-depth (50 ks) pointings covering most of TaurusAuriga (Güdel et al. 2007; and $A \mathscr{G} A$ vol. 468); 169 PMS stellar systems were observed, of which 136 were detected in X-rays.

- Droxo: 'Deep Rho Ophiuchi X-ray Observation' (PI S. Sciortino): a 500 ks deep $X M M-N e w t o n$ pointing of $\rho \mathrm{Oph} ; 111$ sources down to $\log L_{\mathrm{X}}\left[\mathrm{erg} \mathrm{s}^{-1}\right]=28.3$ (Giardino et al. 2007; Pillitteri et al., in prep.).

- $N G C 1893$ (PI G. Micela): a 450 ks deep Chandra observation of the massive SFR NGC 1893, at $d \sim 3 \mathrm{kpc}$ in the anticenter direction, aimed at studying with sufficient 
sensitivity stellar formation and early evolution in the outer part of the Galaxy (Caramazza et al. 2008, in prep.).

- soxs 'Spitzer Orion-A XMM-Newton Survey' (PI S. Wolk): 7 (+2) 50 ks XMMNewton pointings in southern Orion, complemented with new Spitzer data. Data analysis is in progress, with detection of $\sim 1700$ Class I-II young stellar objects, and $\sim 1100$ X-ray sources (Wolk et al. 2009; Pillitteri et al., this volume).

- The Carina Nebula survey (PI L. Townsley): a wide-angle Chandra survey of the giant Carina SFR, with 25 pointings covering among others the clusters Trumpler 16 (with $\eta$ Car), Trumpler 14 and Collinder 228, and overall $>70 \mathrm{O}$ and WR stars.

It is also worth mentioning in this context the recently approved $1 \mathrm{Ms}$ mosaic of Chandra observations of the Cygnus OB2 giant SFR (PI J. Drake), although observations have not yet started. This will undoubtedly provide better insights into this very massive and rich cluster, where more than $1000 \mathrm{X}$-ray sources have already been discovered based on a shallower 100 ks observation (Albacete Colombo et al. 2007; Wright and Drake, this volume).

Globally, these large projects will permit more detailed studies of star formation occurring in very different environments, and at various evolutionary stages. A wealth of new results are likely to come once they are completed.

\section{Acknowledgement}

I acknowledge support from ASI-INAF contract I/088/06/0 (Italy).

\section{References}

Albacete Colombo, J. F., Méndez, M., \& Morrell, N. I. 2003, MNRAS, 346, 704

Albacete Colombo, J. F., Flaccomio, E., Micela, G., Sciortino, S., \& Damiani, F. 2007, A\&A, 464,211

Albacete Colombo, J. F., Damiani, F., Micela, G., Sciortino, S., \& Harnden Jr., F. R. 2008, $A \& A, 490,1055$

Antokhin, I. I., Rauw, G., Vreux, J.-M., van der Hucht, K. A., \& Brown, J. C. 2008, A\&A, 477, 593

Argiroffi, C., Maggio, A., Peres, G., Stelzer, B., \& Neuhäuser, R. 2005, A\&\&A, 439, 1149

Argiroffi, C., Favata, F., Flaccomio, E., Maggio, A., Micela, G., Peres, G., \& Sciortino, S. 2006, $A \& A, 459,199$

Broos, P. S., Feigelson, E. D., Townsley, L. K., Getman, K. V., Wang, J., Garmire, G. P., Jiang, Z., \& Tsuboi, Y. 2007, ApJS, 169, 353

Caramazza, M., Micela, G., Prisinzano, L., Rebull, L., Sciortino, S., \& Stauffer, J. R. 2008, $A \mathscr{E} A, 488,211$

Clark, J. S., Muno, M. P., Negueruela, I., Dougherty, S. M., Crowther, P. A., Goodwin, S. P., \& de Grijs, R. 2008, A\&BA, 477, 147

Currie, T., Evans, N. R., Spitzbart, B. D., Irwin, J., Wolk, S. J., Hernandez, J., Kenyon, S. J., \& Pasachoff, J. M. 2009, AJ, 137, 3210

Damiani, F., Micela, G., Sciortino, S., \& Harnden Jr., F. R. 1995, ApJ, 446, 331

Damiani, F., Flaccomio, E., Micela, G., Sciortino, S., Harnden Jr., F. R., \& Murray, S. S. 2004, ApJ, 608, 781

Damiani, F., Micela, G., Sciortino, S., Huélamo, N., Moitinho, A., Harnden Jr., F. R., \& Murray, S. S. $2006, A \mathscr{E} A, 460,133$

Damiani, F., Micela, G., Sciortino, S., \& Harnden Jr., F. R. 2009, AIP Conf. Ser., 1094, 916

De Becker, M., Rauw, G., Blomme, R., Pittard, J. M., Stevens, I. R., \& Runacres, M. C. 2005, $A \mathscr{E} A, 437,1029$

Delgado, A. J., González-Martín, O., Alfaro, E. J., \& Yun, J. 2006, ApJ, 646, 269

Evans, N. R., Seward, F. D.; Krauss, M. I., Isobe, T., Nichols, J., Schlegel, E. M., \& Wolk, S. J. 2003, ApJ, 589, 509 
Evans, N. R., Schlegel, E. M., Waldron, W. L., Seward, F. D., Krauss, M. I., Nichols, J., \& Wolk, S. J. 2004, ApJ, 612, 1065

Ezoe, Y., Kokubun, M., Makishima, K., Sekimoto, Y., \& Matsuzaki, K. 2006, ApJ, 638, 860

Ezoe, Y., Kokubun, M., Makishima, K., Sekimoto, Y., \& Matsuzaki, K. 2006, ApJ (Letters), $649, \mathrm{~L} 123$

Ezoe, Y., Hamaguchi, K., Gruendl, R. A., Chu, Y.-H., Petre, R., \& Corcoran, M. F. 2009, PASJ, $61, \mathrm{~S} 123$

Favata, F., Giardino, G., Micela, G., Sciortino, S., \& Damiani, F. 2003, A\&\&A, 403, 187

Feigelson, E. D. \& Montmerle, T. 1999, ARA\&A, 37, 363

Feigelson, E. D., Gaffney III, J. A., Garmire, G., Hillenbrand, L. A., \& Townsley, L. 2003, ApJ, 584,911

Feigelson, E. D., Lawson, W. A., \& Garmire, G. P. 2003, ApJ, 599, 1207

Feigelson, E. D. \& Lawson, W. A. 2004, ApJ, 614, 267

Feigelson, E., Townsley, L., Güdel, M., \& Stassun, K. 2007, in: Reipurth, B., Jewitt, D., \& Keil, K., Protostars and Planets V, p. 313 (Tucson: University of Arizona Press)

Feigelson, E. D. \& Townsley, L. K. 2008, ApJ, 673, 354

Feigelson, E. D., Martin, A. L., McNeill, C. J., Broos, P. S., \& Garmire, G. P. 2009, AJ, 138, 227

Flaccomio, E., Damiani, F., Micela, G., Sciortino, S., Harnden Jr., F. R., Murray, S. S., \& Wolk, S. J. 2003, ApJ, 582, 382

Flaccomio, E., Damiani, F., Micela, G., Sciortino, S., Harnden Jr., F. R., Murray, S. S., \& Wolk, S. J. 2003, ApJ, 582, 398

Flaccomio, E., Micela, G., \& Sciortino, S. 2006, A\& $A, 455,903$

Forbrich, J., Preibisch, T., \& Menten, K. M. 2006, A\&A, 446, 155

Forbrich, J. \& Preibisch, T. 2007, A\&A, 475, 959

Franciosini, E., Pallavicini, R., \& Sanz-Forcada, J. 2006, A\& A, 446, 501

Gagné, M., Skinner, S. L., \& Daniel, K. J. 2004, ApJ, 613, 393

Garmire, G., Feigelson, E. D., Broos, P., Hillenbrand, L. A., Pravdo, S. H., Townsley, L., \& Tsuboi, Y. 2000, AJ, 120, 1426

Getman, K. V., Feigelson, E. D., Townsley, L., Bally, J., Lada, C. J., \& Reipurth, B. 2002, ApJ, 575,354

Getman, K. V., et al. 2005, ApJS, 160, 319

Getman, K. V., Feigelson, E. D., Townsley, L., Broos, P., Garmire, G., \& Tsujimoto, M. 2006, ApJS, 163, 306

Getman, K. V., Feigelson, E. D., Garmire, G., Broos, P., \& Wang, J. 2007, ApJ, 654, 316

Getman, K. V., Feigelson, E. D., Lawson, W. A., Broos, P. S., \& Garmire, G. P. 2008, ApJ, 673, 331

Getman, K. V., Feigelson, E. D., Luhman, K. L., Sicilia-Aguilar, A., Wang, J., \& Garmire, G. P. 2009, ApJ, 699, 1454

Giardino, G., Favata, F., \& Micela, G. 2004, A\&A, 424, 965

Giardino, G., Favata, F., Pillitteri, I., Flaccomio, E., Micela, G., \& Sciortino, S. 2007, A\&A, 475,891

Giardino, G., Favata, F., Micela, G., Sciortino, S., \& Winston, E. 2007, A\&A A, 463, 275

Gizis, J. E. \& Bharat, R. 2004, ApJ (Letters), 608, L113

Gondoin, P. 2006, A\& A, 454, 595

Grosso, N., Montmerle, T., Feigelson, E. D., \& Forbes, T. G. 2004, A\& A, 419, 653

Guarcello, M. G., Prisinzano, L., Micela, G., Damiani, F., Peres, G., \& Sciortino, S. 2007, $A \& A$, 462,245

Güdel, M. 2004, A\&A Rev., 12, 71

Güdel, M., et al. 2007, A\&A, 468, 353

Güdel, M., Briggs, K. R., Montmerle, T., Audard, M., Rebull, L., \& Skinner, S. L. 2008, Science, 319,309

Güdel, M. \& Nazé, Y. 2009, A\&A Rev., 17, 309

Hamaguchi, K., Corcoran, M. F., Petre, R., White, N. E., Stelzer, B., Nedachi, K., Kobayashi, N., \& Tokunaga, A. T. 2005, ApJ, 623, 291 
Hofner, P., Delgado, H., Whitney, B., Churchwell, E., \& Linz, H. 2002, ApJ (Letters), 579, L95 Imanishi, K., Koyama, K., \& Tsuboi, Y. 2001, ApJ, 557, 747

Jeffries, R. D., Evans, P. A., Pye, J. P., \& Briggs, K. R. 2006, MNRAS, 367, 781

Jeffries, R. D., Naylor, T., Walter, F. M., Pozzo, M. P., \& Devey, C. R. 2009, MNRAS, 393, 538

Kastner, J. H., Huenemoerder, D. P., Schulz, N. S., Canizares, C. R., \& Weintraub, D. A. 2002, ApJ, 567, 434

Kohno, M., Koyama, K., \& Hamaguchi, K. 2002, ApJ, 567, 423

Koo, B.-C., Lee, J.-J., Seward, F. D., \& Moon, D.-S. 2005, ApJ, 633, 946

Lada, C. J., Gottlieb, C. A., Gottlieb, E. W., \& Gull, T. R. 1976, ApJ, 203, 159

Lefloch, B. \& Lazareff, B. 1994, A\&A, 289, 559

Linder, N., Rauw, G., Manfroid, J., Damerdji, Y., De Becker, M., Eenens, P., Royer, P., \& Vreux, J.-M. 2009, A\&\&A, 495, 231

Linsky, J. L., Gagné, M., Mytyk, A., McCaughrean, M., \& Andersen, M. 2007, ApJ, 654, 347

Mamajek, E. E., Lawson, W. A., \& Feigelson, E. D. 1999, ApJ (Letters), 516, L77

Marino, A., Micela, G., Peres, G., Pillitteri, I., \& Sciortino, S. 2005, A\& A, 430, 287

Moffat, A. F. J., et al. 2002, ApJ, 573, 191

Moitinho, A., Alves, J., Huélamo, N., \& Lada, C. J. 2001, ApJ (Letters), 563, L73

Muno, M. P., Law, C., Clark, J. S., Dougherty, S. M., de Grijs, R., Portegies Zwart, S., \& Yusef-Zadeh, F. 2006, ApJ, 650, 203

Muno, M. P., Gaensler, B. M., Clark, J. S., de Grijs, R., Pooley, D., Stevens, I. R., \& Portegies Zwart, S. F. 2007, MNRAS, 378, L44

Nakajima, H., Imanishi, K., Takagi, S.-I., Koyama, K., \& Tsujimoto, M. 2003, PASJ, 55, 635

Nazé, Y., Rauw, G., \& Manfroid, J. 2008, A\&GA, 483, 171

Osten, R. A. \& Wolk, S. J. 2009, ApJ, 691, 1128

Ozawa, H., Grosso, N., \& Montmerle, T. 2005, A\& $A, 438,661$

Pallavicini, R., Golub, L., Rosner, R., Vaiana, G. S., Ayres, T., \& Linsky, J. L. 1981, ApJ, 248, 279

Poteet, C., Marchenko, S., Corcoran, M., \& Andersen, M. 2004, BAAS, 36, 1380

Pravdo, S. H., Tsuboi, Y., Suzuki, Y., Thompson, T. J., \& Rebull, L. 2009, ApJ, 690, 850

Preibisch, T. \& Zinnecker, H. 2001, AJ, 122, 866

Preibisch, T. \& Zinnecker, H. 2002, AJ, 123, 1613

Preibisch, T. 2003, A\& $A, 401,543$

Preibisch, T. 2003, $A \mathscr{E} A$, 410, 951

Preibisch, T. \& Zinnecker, H. 2004, A\& A, 422, 1001

Prisinzano, L., Damiani, F., Micela, G., \& Sciortino, S. 2005, A\&A, 430, 941

Prisinzano, L., Damiani, F., Micela, G., \& Pillitteri, I. 2007, A\& $A, 462,123$

Ramírez, S. V., Rebull, L., Stauffer, J., Hearty, T., Hillenbrand, L., Jones, B., Makidon, R., Pravdo, S., Strom, S., \& Werner, M. 2004, AJ, 127, 2659

Ramírez, S. V., Rebull, L., Stauffer, J., Strom, S., Hillenbrand, L., Hearty, T., Kopan, E. L. Pravdo, S., Makidon, R., \& Jones, B. 2004, AJ, 128, 787

Randich, S. 2000, ASP Conf. Ser., 198, 401

Rauw, G., Nazé, Y.; Gosset, E., Stevens, I. R., Blomme, R., Corcoran, M. F., Pittard, J. M., \& Runacres, M. C. 2002, A\& A, 395, 499

Rauw, G., De Becker, M., Gosset, E., Pittard, J. M., \& Stevens, I. R. 2003, A\& $A, 407,925$

Rebull, L. M., Stauffer, J. R., Ramírez, S. V., Flaccomio, E., Sciortino, S., Micela, G., Strom, S. E., \& Wolff, S. C. 2006, AJ, 131, 2934

Rho, J., Ramírez, S. V., Corcoran, M. F., Hamaguchi, K., \& Lefloch, B. 2004, ApJ, 607, 904

Robrade, J. \& Schmitt, J. H. M. M. 2007, A\&A A, 461, 669

Sana, H., Rauw, G., Nazé, Y., Gosset, E., \& Vreux, J.-M. 2006, MNRAS, 372, 661

Sana, H., Rauw, G., Sung, H., Gosset, E., \& Vreux, J.-M. 2007, MNRAS, 377, 945

Scelsi, L., Sacco, G., Affer, L., Argiroffi, C., Pillitteri, I., Maggio, A., \& Micela, G. 2008, A\&A, 490, 601

Simon, T., Andrews, S. M., Rayner, J. T., \& Drake, S. A. 2004, ApJ, 611, 940

Simon, T. \& Dahm, S. E. 2005, ApJ, 618, 795

Simon, T. 2006, AJ, 131, 501 
Simon, T. 2009, ApJ, 694, 425

Skinner, S., Gagné, M., \& Belzer, E. 2003, ApJ, 598, 375

Skinner, S. L., Zhekov, S. A., Palla, F., \& Barbosa, C. L. D. R. 2005, MNRAS, 361, 191

Skinner, S. L., Simmons, A. E., Zhekov, S. A., Teodoro, M., Damineli, A., \& Palla, F. 2006, ApJ (Letters), 639, L35

Skinner, S. L., Simmons, A. E., Audard, M., \& Güdel, M. 2007, ApJ, 658, 1144

Skinner, S. L., Sokal, K. R., Cohen, D. H., Gagné, M., Owocki, S. P., \& Townsend, R. D. 2008, ApJ, 683, 796

Skinner, S. L., Sokal, K. R., Güdel, M., \& Briggs, K. R. 2009, ApJ, 696, 766

Skinner, S. L., Sokal, K. R., Megeath, S. T., Güdel, M., Audard, M., Flaherty, K. M., Meyer, M. R., \& Damineli, A. 2009, ApJ, 701, 710

Stelzer, B. \& Schmitt, J. H. M. M. 2004, A\& A, 418, 687

Stelzer, B., Micela, G., \& Neuhäuser, R. 2004, A\&A, 423, 1029

Stelzer, B. \& Scholz, A. 2009, A\&SA, 507, 227

Sung, H., Bessell, M. S., \& Lee, S.-W. 1998, AJ, 115, 734

Takagi, S.-I., Murakami, H., \& Koyama, K. 2002, ApJ, 573, 275

Testa, P., Huenemoerder, D. P., Schulz, N. S., \& Ishibashi, K. 2008, ApJ, 687, 579

Townsley, L. K., Feigelson, E. D., Montmerle, T., Broos, P. S., Chu, Y.-H., \& Garmire, G. P. 2003, ApJ, 593, 874

Townsley, L. K., Broos, P. S., Feigelson, E. D., Brandl, B. R., Chu, Y.-H., Garmire, G. P., \& Pavlov, G. G. 2006, AJ, 131, 2140

Townsley, L. K. 2006, in: Livio M. (ed.), Massive Stars: From Population III and GRBs to the Milky Way, in press (astro-ph/0608173)

Tsuboi, Y., Koyama, K., Hamaguchi, K., Tatematsu, K., Sekimoto, Y., Bally, J., \& Reipurth, B. $2001, A p J, 554,734$

Tsujimoto, M., Koyama, K., Tsuboi, Y., Goto, M., \& Kobayashi, N. 2002, ApJ, 566, 974

Tsujimoto, M., Kobayashi, N., \& Tsuboi, Y. 2005, AJ, 130, 2212

Tsujimoto, M., Hosokawa, T., Feigelson, E. D., Getman, K. V., \& Broos, P. S. 2006, ApJ, 653, 409

Tsujimoto, M., et al. 2007, ApJ, 665, 719

Walter, F. M., Wolk, S. J., Freyberg, M., \& Schmitt, J. H. M. M. 1997, Mem. SAIt, 68, 1081

Wang, Q. D., Dong, H., \& Lang, C. 2006, MNRAS, 371, 38

Wang, J., Townsley, L. K., Feigelson, E. D., Getman, K. V., Broos, P. S., Garmire, G. P., \& Tsujimoto, M. 2007, ApJS, 168, 100

Wang, J., Townsley, L. K., Feigelson, E. D., Broos, P. S., Getman, K. V., Román-Zúñiga, C. G., \& Lada, E. 2008, ApJ, 675, 464

Wang, J., Feigelson, E. D., Townsley, L. K., Román-Zúñiga, C. G., Lada, E., \& Garmire, G. 2009, ApJ, 696, 47

Winston, E., Megeath, S. T., Wolk, S. J., Muzerolle, J., Gutermuth, R., Hora, J. L., Allen, L. E., Spitzbart, B., Myers, P., \& Fazio, G. G. 2007, ApJ, 669, 493

Wolk, S. J., Bourke, T. L., Smith, R. K., Spitzbart, B., \& Alves, J. 2002, ApJ (Letters), 580, L161

Wolk, S. J., Spitzbart, B. D., Bourke, T. L., \& Alves, J. 2006, AJ, 132, 1100

Wolk, S. J., Spitzbart, B. D., Bourke, T. L., Gutermuth, R. A., Vigil, M., \& Comerón, F. 2008, AJ, 135,693

Wolk, S. J. 2009, AIP Conf. Ser., 1094, 959

Yusef-Zadeh, F., Law, C., Wardle, M., Wang, Q. D., Fruscione, A., Lang, C. C., \& Cotera, A. 2002, ApJ, 570, 665 